\title{
MEASURE OF NONHYPERCONVEXITY AND FIXED-POINT THEOREMS
}

\author{
DARIUSZ BUGAJEWSKI AND RAFAEL ESPÍNOLA
}

Received 6 November 2001

The aim of this paper is to work with the measure of nonhyperconvexity in a similar way as J. Cano (1990) does with the index of nonconvexity. We apply this measure to obtain different extensions of the famous Schauder fixed-point theorem in hyperconvex spaces.

\section{Introduction}

In this paper, we work with the notion of the measure of nonhyperconvexity introduced by Cianciaruso and De Pascale [6] in order to obtain new fixed-point theorems in hyperconvex metric spaces. This class of metric spaces was introduced by Aronszajn and Panitchpakdi [1] in 1956 to study problems on extension of uniformly continuous mappings. Several and interesting properties of these spaces were shown in Aronszajn and Panitchpakdi's original paper, some of these properties turned out to be crucial in the successful searching for fixedpoint theorems in hyperconvex metric spaces. More precisely, this research began when Sine [13] and Soardi [14] independently proved in 1979 that bounded hyperconvex metric spaces have the fixed-point property for nonexpansive mappings. Since then, authors like J. B. Baillon, M. A. Khamsi, W. A. Kirk, S. Park, G. Yuan, and many others, including both authors of this paper, have been attracted by this subject and have contributed to a wide development of it (for a recent survey see [11, Chapter 13]).

Hyperconvex metric spaces can be defined as follows: given two metric spaces $(Y, d)$ and $(X, \rho)$, we say that a mapping $T: Y \rightarrow X$ is nonexpansive if $\rho(T x, T y) \leq$ $d(x, y)$ for any $x$ and $y$ in $Y$. The pair $(Y, X)$ is said to have the extension property for nonexpansive mappings, if every nonexpansive mapping from an arbitrary subset $S$ of $Y$ into $X$ can be extended as a nonexpansive mapping to the whole $Y$ into $X$. A metric space $X$ is said to be hyperconvex if the pair $(Y, X)$ 


\section{Measure of nonhyperconvexity and fixed-point theorems}

enjoys the extension property for nonexpansive mappings for any metric space $Y$.

Hyperconvex spaces have a good number of properties that is, for instance, any hyperconvex metric space is complete. The simplest example of hyperconvex spaces are the finite-dimensional real spaces endowed with the maximum norm. Other simple examples of hyperconvex metric spaces are provided by closed balls and intersection of closed balls of the above spaces endowed with the induced metric. For a complete treatment of these and other results on hyperconvexity, as well as for a more general study of hyperconvex metric spaces, the reader may consult the recent overview on hyperconvexity and fixed-point theory developed in [11, Chapter 13].

Cano [5] stated a new version of the well-known Schauder fixed-point theorem relaxing the condition on the convexity of the set by using the so-called measure of nonconvexity introduced by Eisenfeld and Lakshmikantham [7] in 1976. The aim of this paper is to work with a similar concept which measures the lack of hyperconvexity of a metric space, in order to sharpen, among others, Schauder and Darbo-Sadovski's fixed-point theorems in hyperconvex spaces. In Section 2, the authors give an equivalent definition for the measure of nonhyperconvexity, introduced by Cianciaruso and De Pascale in [6], which will turn out to be more convenient for the kind of problems we are concerned with. Section 3 is devoted to obtain new fixed-point theorems under certain hyperconvex hypothesis regarding different compactness conditions on the mapping which are closely related to recent results on hyperconvex spaces.

\section{Measure of nonhyperconvexity}

Hyperconvex hulls of metric spaces are needed to define the measure of nonhyperconvexity. Isbell introduced the concept of a hyperconvex hull in his very celebrated paper [10] in the following way. Let $X$ be a metric space. The pair $(E, e)$, where $E$ is a hyperconvex space and $e$ is an isometric embedding of $X$ in $E$, is called a hyperconvex hull of the metric space $X$ if no hyperconvex proper subset of $E$ contains $e(X)$.

In particular, Isbell proved that for any metric space $X$ there exists a natural hyperconvex hull which we will denote by $(\varepsilon(X), e)$, and that, although a hyperconvex hull need not be uniquely determined, any two hyperconvex hulls are isometric. This concept has been of great importance in order to obtain topological fixed-point theorems in hyperconvex spaces (see, e.g., $[4,9,8,11]$ ). The following definition was given by Cianciaruso and De Pascale in [6].

Definition 2.1. Let $X$ be a metric space and $(\varepsilon(X), e)$ its natural hyperconvex hull. Then, the measure of nonhyperconvexity of $X$ is given by

$$
\mu(X)=H(e(X), \varepsilon(X)),
$$

where $H(\cdot, \cdot)$ stands for the Hausdorff distance between subsets of a metric 
space, that is, in this case $H(e(X), \varepsilon(X))=\sup \{d(y, e(X)): y \in \varepsilon(X)\}$, where $d(\cdot, \cdot)$ is a distance between a point and a set.

The goal of this section is to attract the attention to the fact that the measure of nonhyperconvexity of a metric space can be defined in a less rigid way. This is provided by the following theorem.

Theorem 2.2. Let $X$ be a metric space such that (E,id), where id stands for the identity map on $X$, is a hyperconvex hull of $X$, then

$$
\mu(X)=H(X, E)
$$

Proof. We only need to prove that $H(X, E)=H(e(X), \varepsilon(X))$, where $(\varepsilon(X), e)$ is the natural hyperconvex hull of $X$. However, this immediately follows from the fact, proved by Isbell in [10], that there exists an isometry $i: E \rightarrow \varepsilon(X)$ such that $i(X)=e(X)$.

Now, if $X$ is a metric space, then there exists a hyperconvex space $M$ such that $X \subseteq M$. It is not hard to observe (see [11, Chapter 13] for details) that there exists such a hyperconvex hull $(h(X)$, id) of $X$ as in the statement of the theorem. From now on, given a metric space $X$ and $A \subseteq X, h(A)$ will stand for a hyperconvex hull of $A$ as above. Notice that, in this way we avoid to explicitly deal with the hyperconvex space $M$. Notice also that, in particular, $\mu(A)=H(A, h(A))$.

The following proposition states a very important property of the measure of nonhyperconvexity. The proof of this fact, as well as further properties of this measure, can be found in [6].

Proposition 2.3. Let $X$ be a complete metric space. Then $\mu(X)=0$ if and only if $X$ is hyperconvex.

\section{Fixed-point theorems}

In this section, we deal with topological fixed-point theorems (i.e., Schauder and Darbo-Sadovski type theorems). Our aim is to relax some of the hypothesis given in some hyperconvex versions of these theorems by using the measure of nonhyperconvexity. As a beginning, we introduce some notions.

Definition 3.1. Let $X$ be a metric space. Then a mapping $f: X \rightarrow X$ is said to be $\mu$-contractive if

$$
\liminf _{n \rightarrow \infty} \mu\left(f^{n}(X)\right)=0
$$

where $f^{n}$ stands for the $n$th iterate of $f$.

The following definitions have been widely studied, the reader may consult [2] or [11, Chapter 8] for recent treatments on them. 
114 Measure of nonhyperconvexity and fixed-point theorems

Definition 3.2. Let $A$ be a subset of a metric space $X$. Then the Kuratowski measure of noncompactness of $A, \alpha(A)$, is defined as

$$
\alpha(A)=\inf \left\{\varepsilon: A \subset \bigcup_{i=1}^{n(\varepsilon)} A_{i} \text { with } \operatorname{diam} A_{i} \leq \varepsilon\right\}
$$

Definition 3.3. Let $X$ be a metric space. A mapping $f: X \rightarrow X$ is $\alpha$-contractive if

$$
\inf \left\{\alpha\left(f^{n}(X)\right): n \in \mathbb{N}\right\}=0 \text {. }
$$

Theorem 3.4 is a version of the Schauder fixed-point theorem under hyperconvex hypothesis.

Theorem 3.4. Let $C$ be a nonempty compact metric space and let $f: C \rightarrow C$ be a continuous and $\mu$-contractive mapping. Then $f$ has a fixed-point in $C$.

Proof. Consider the set $A=\bigcap_{n=1}^{\infty} f^{n}(C)$. Obviously, $A$ is a nonempty and compact subset of $C$ such that $f(A) \subset A$. Furthermore, we have $H(A, h(A)) \leq H(A$, $\left.h\left(f^{n}(C)\right)\right)$ for any $n \in \mathbb{N}$. But $H\left(A, h\left(f^{n}(C)\right)\right) \leq H\left(A, f^{n}(C)\right)+H\left(f^{n}(C)\right.$, $\left.h\left(f^{n}(C)\right)\right)$ for every $n$, so, passing to a subsequence if necessary and applying the fact that $f^{n}(C)$ is compact for every $n$, we deduce that $H(A, h(A))=0$. Since $A$ is closed, it is hyperconvex. Hence, $f$ has a fixed-point in $A$.

In connection with this theorem, we also have the following one.

TheOrem 3.5. Let $C$ be a nonempty compact metric space and let $f: C \rightarrow C$ be a continuous mapping such that $\mu\left(f^{n_{0}}(C)\right)=0$ for a certain $n_{0} \in \mathbb{N}$. Then $f$ has a fixed-point in $C$.

Proof. If for some $n_{0} \in \mathbb{N}, \mu\left(f^{n_{0}}(C)\right)=0$, then, by Proposition 2.3, $f^{n_{0}}(C)$ is hyperconvex as well as, in this case, compact. Hence, by the Schauder fixed-point in hyperconvex spaces, $f$ has a fixed-point in $f^{n_{0}}(C)$.

The following lemma leads to an extension of Theorem 3.4.

LEMMA 3.6. Let $\left\{K_{i}\right\}_{i=1}^{\infty}$ be a sequence of nonempty bounded and closed subsets of a metric space $X$ such that $K_{i+1} \subset K_{i}$ for $i \in \mathbb{N}$ and $\liminf _{i \rightarrow \infty} \mu\left(K_{i}\right)=0$. Then $\bigcap_{i=1}^{\infty} K_{i}$ is hyperconvex and

$$
\bigcap_{i=1}^{\infty} K_{i}=\bigcap_{i=1}^{\infty} h\left(K_{i}\right) \neq \varnothing
$$

for any possible option of the hyperconvex hulls $h\left(K_{i}\right)$. 
Proof. In order to prove that $\bigcap_{i=1}^{\infty} K_{i}$ is nonempty and hyperconvex, it is enough, by Baillon's intersecting theorem [3], to prove that it coincides with the intersection of a decreasing family of nonempty bounded hyperconvex sets. However, it is not hard to see that the sequence of hyperconvex hulls $\left(h\left(K_{i}\right)\right)$ may be chosen, so it is also a descending sequence of sets (see [11, Chapter 13] for details). Hence, to complete this part of the proof, it suffices to prove that

$$
\bigcap_{i=1}^{\infty} K_{i}=\bigcap_{i=1}^{\infty} h\left(K_{i}\right),
$$

where the sequence of hyperconvex hulls has been fixed as above.

The inclusion " $\subseteq$ " is trivial. In order to prove the other inclusion, let $\varepsilon>0$ and $x \in \bigcap_{i=1}^{\infty} h\left(K_{i}\right)$. Then $B(x, \varepsilon) \cap K_{i} \neq \varnothing$ for every $i$ such that $\mu\left(K_{i}\right)<\varepsilon$, where $B(x, \varepsilon)$ stands for the closed ball of center $x$ and radius $\varepsilon$. Moreover, since $K_{i+1} \subset$ $K_{i}, B(x, \varepsilon)$ contains points of each set $K_{i}$. Thus, since $K_{i}$ is closed, $x \in K_{i}$ for every $i \in \mathbb{N}$. Hence, $x \in \bigcap_{i=1}^{\infty} K_{i}$ and the claim is proved.

To finish the proof, it suffices to prove the equality between the intersections for the case when the sequence of hyperconvex hulls is not a decreasing one. But, since $\bigcap_{i=1}^{\infty} K_{i} \neq \varnothing$, we also have that $\bigcap_{i=1}^{\infty} h\left(K_{i}\right) \neq \varnothing$. The proof follows just as above.

The following theorem improves Theorem 3.4 as it is not required for the metric space to be compact. Notice also that any mapping, under the hypothesis of Theorem 3.4, must also satisfy the hypothesis of the next theorem.

Theorem 3.7. Let $A$ be a nonempty bounded and complete metric space, and let $f: A \rightarrow A$ be a continuous and both $\alpha$-and $\mu$-contractive. Then $f$ has a fixed-point.

Proof. Since $f$ is $\mu$-contractive, we may apply Lemma 3.6 to deduce that the set $L=\bigcap_{n=0}^{\infty} \overline{f^{n}(A)}$ is a nonempty hyperconvex set. Moreover, from the $\alpha$-contractiveness of $f$ and the very well-known property that the Kuratowski measure of noncompactness is monotone (see [11, Chapter 8] for details), $L$ is compact. Hence, $L$ is an $f$-invariant compact hyperconvex set and so $f$ has a fixed-point in $L$.

In what follows, we state different variants of Theorem 3.7. The next is related to the theory of limit compact mappings. These mappings were introduced by Sadovskiu in [12], in order to obtain new versions of Schauder's theorem. The hyperconvex version of those mappings was first studied in [8], see also [11, Chapter 13].

Definition 3.8. Let $X$ be a metric space and $f: X \rightarrow X$ a mapping. The $\gamma$ transfinite iterates of $X$ through $f$ are defined as

$$
f\left(f^{\gamma-1}(X)\right) \text { if } \gamma \text { is an ordinal with antecedent }
$$


116 Measure of nonhyperconvexity and fixed-point theorems

or

$$
\bigcap_{\beta<\gamma} f^{\beta}(X) \text { if } \gamma \text { is a limit ordinal. }
$$

Definition 3.9. Let $X$ be a metric space. Then a mapping $f: X \rightarrow X$ is said to be an extended $\mu$-contractive mapping if

$$
\liminf _{\gamma} \mu\left(f^{\gamma}(X)\right)=0 .
$$

(We understand here that $\mu(\varnothing)=+\infty$.)

The mapping $f$ is said to be an extended $\alpha$-contractive mapping if

$$
\inf \left\{\alpha\left(f^{\gamma}(X)\right): \gamma \text { is ordinal number with } f^{\gamma}(X) \neq \varnothing\right\}=0 \text {. }
$$

Note that the transfinite sequence of the $\gamma$-iterates of $X$ through $f$ is eventually constant (either as the empty set or as a nonempty set) from a certain ordinal number on. The following lemma is analogous to Lemma 3.6 in this transfinite setting. We omit the proof since the argument to follow is not very different from that of Lemma 3.6.

Lemma 3.10. Let $\left\{K_{\gamma}\right\}$ be a descending transfinite sequence of nonempty bounded closed subsets of a metric space $X$ such that $\liminf _{\gamma} \mu\left(K_{\gamma}\right)=0$. Then $\bigcap K_{\gamma}$ is hyperconvex and

$$
\bigcap K_{\gamma}=\bigcap h\left(K_{\gamma}\right) \neq \varnothing \text {. }
$$

This lemma allows us to give an analogous theorem to Theorem 3.7 for extended contractive mappings.

THEOREM 3.11. Let $A$ be a nonempty bounded and complete metric space, and let $f: A \rightarrow A$ be a continuous and both extended $\alpha$-contractive and extended $\mu$ contractive mapping. Then $f$ has a fixed-point.

Proof. The proof of this theorem follows introducing small changes in that of Theorem 3.7.

The next theorem takes place in the linear context since it is an extension of those theorems treated by Cano in [5], where the author worked with the concept of measure of nonconvexity. If $A$ is a subset of a linear metric space, then the measure of nonconvexity $\beta(A)$ of $A$ is given by

$$
\beta(A)=H(A, \operatorname{conv} A),
$$

where $H$ is again the Hausdorff distance and $\operatorname{conv} A$ stands for the convex hull of $A$. 
Definition 3.12. Let $A$ be a subset of a linear metric space. Then a mapping $f$ : $A \rightarrow A$ is said to be $\beta$-contractive if

$$
\liminf _{n \rightarrow \infty} \beta\left(f^{n}(A)\right)=0
$$

THEOREM 3.13. Let $A$ be a nonempty and closed subset of a Banach space, and let $f: A \rightarrow A$ be a continuous and $\beta$-contractive mapping. If there exists a point $x_{0}$ in A such that the implication

$$
(V=\operatorname{conv} f(V)) \quad \text { or } \quad\left(V=f(V) \cup\left\{x_{0}\right\}\right) \Longrightarrow V \text { is relatively compact }
$$

holds for every subset $V$ of $A$, then $f$ has a fixed-point.

Proof. Arguing similarly as in [15], we infer that there exists a set $Z \subset A$ such that $f(Z)=Z$. Let $D=\bigcap_{n=0}^{\infty} \overline{f^{n}(A)}$. Obviously, $f(D) \subset D$ and $Z \subset D$. By Cano's lemma from [5], $D$ is convex. Let $R(X)=\operatorname{conv} f(X)$ for $X \subset A$ and let $\Omega$ denote the family of all subsets $X$ of $A$ such that $Z \subset X$ and $R(X) \subset X$. Since $Z \subset D$ and conv $f(D) \subset D$, so $\Omega$ is nonempty. Denote by $V$ the intersection of all subsets of the family $\Omega$. As $Z \subset V, V$ is nonempty and $Z=f(Z) \subset R(Z) \subset R(V)$. Since $R(V) \subset R(X) \subset X$ for all $X \in \Omega, R(V) \subset V$, and therefore $V \in \Omega$. Moreover, $R(R(V)) \subset R(V)$, and therefore $R(V) \in \Omega$. Consequently, $V=R(V)$, that is, $V=\operatorname{conv} f(V)$. In view of (3.13), this implies that $\bar{V}$ is a compact subset of $D$. Now the Schauder fixed-point theorem implies that $f$ has a fixed-point in $\bar{V}$.

Theorem 3.13 extends [15, Theorem 1]. Note that a mapping $f$ satisfying (3.13) does not have to be $\alpha$-contractive as the following example shows.

Example 3.14. Let $X=\left\{x=\left(x_{n}\right) \in l_{\infty}: 0 \leq x_{n} \leq 1\right.$ for $\left.n \in \mathbb{N}\right\}$. Define

$$
f(x)=\left(0, \sqrt{x_{1}}, \sqrt{x_{2}}, \ldots\right), \quad x \in X
$$

It can be shown that $f$ satisfies (3.13) but still $\alpha\left(f^{n}(X)\right)=1$ for each $n \in \mathbb{N}$ (see [4, Example 4]).

We finish this paper with the following theorem which is an extension of [4, Theorem 3].

Theorem 3.15. Let $A$ be a nonempty complete metric space, and let $f: A \rightarrow A$ be a continuous and $\mu$-contractive mapping. Suppose that there exists a point $x_{0}$ in $A$ such that for $V \subset A$, the equality $f(V) \cup\left\{x_{0}\right\}=V$ implies the relative compactness of $V$, and that only relatively compact sets $V$ can be equal to $h(f(V))$. Then $f$ has a fixed-point. 
Proof. Again let $Z$ be a subset of $A$ such that $f(Z)=Z$ and let $D=\bigcap_{n=0}^{\infty} \overline{f^{n}(A)}$. Obviously, $f(D) \subset D$ and $Z \subset D$. By Lemma 3.6, it is hyperconvex. Denote by $\Omega$ the family of all sets $H \subset A$ such that $Z \subset H, H$ is hyperconvex and $f(H) \subset H$. Obviously, the family $\Omega$ is nonempty because $D \in \Omega$. Further, we argue similarly as in [4, Theorem 3].

Remark 3.16. Although Cano's paper [5] is the actual motivation for this paper, the definitions of $\beta$-contractive and $\mu$-contractive mappings given here do not quite fit that one of $\beta$-contractive mapping originally given by Cano. In fact, as Cano defined it, a mapping $f: X \rightarrow X$ is said to be $\beta$-contractive if $\inf \left\{\mu\left(f^{n}(X)\right): n \in \mathbb{N}\right\}=0$. Note that since the measure of nonconvexity, as well as the measure of nonhyperconvexity, is nonmonotone, both definitions of $\beta$ contractive mappings are different. Gaps in the proof of Theorem 2 in Cano's paper made us redefine the notion of $\beta$-contractive mapping. Notice also that [5, Theorem 2] becomes doubtless true under this new definition.

\section{Acknowledgment}

The second author was supported by DGICYT, project no. PB96-1338-C01.

\section{References}

[1] N. Aronszajn and P. Panitchpakdi, Extension of uniformly continuous transformations and hyperconvex metric spaces, Pacific J. Math. 6 (1956), 405-439.

[2] J. M. Ayerbe Toledano, T. Domínguez Benavides, and G. López Acedo, Measures of Noncompactness in Metric Fixed Point Theory, Operator Theory: Advances and Applications, vol. 99, Birkhäuser Verlag, Basel, 1997.

[3] J.-B. Baillon, Nonexpansive mapping and hyperconvex spaces, Fixed Point Theory and Its Applications (Berkeley, Calif, 1986) (R. F. Brown, ed.), Contemporary Mathematics, vol. 72, American Mathematical Society, Rhode Island, 1988, pp. 11-19.

[4] D. Bugajewski and E. Grzelaczyk, A fixed point theorem in hyperconvex spaces, Arch. Math. (Basel) 75 (2000), no. 5, 395-400.

[5] J. Cano, A measure of nonconvexity and another extension of Schauder's theorem, Bull. Math. Soc. Sci. Math. R. S. Roumanie (N.S.) 34(82) (1990), no. 1, 3-6.

[6] F. Cianciaruso and E. De Pascale, The Hausdorff measure of non-hyperconvexity, Atti Sem. Mat. Fis. Univ. Modena 47 (1999), no. 1, 261-267.

[7] J. Eisenfeld and V. Lakshmikantham, On a measure of nonconvexity and applications, Yokohama Math. J. 24 (1976), no. 1-2, 133-140.

[8] R. Espínola and G. López, Ultimately compact operators in hyperconvex metric spaces, Nonlinear Analysis and Convex Analysis (Niigata, 1998), World Scientific Publishing, New Jersey, 1999, pp. 142-149.

[9] R. Espinola-Garcia, Darbo-Sadovski's theorem in hyperconvex metric spaces, Rend. Circ. Mat. Palermo (2) Suppl. (1996), no. 40, 129-137.

[10] J. R. Isbell, Six theorems about injective metric spaces, Comment. Math. Helv. 39 (1964), 65-76.

[11] W. A. Kirk and B. Sims (eds.), Handbook of Metric Fixed Point Theory, Kluwer Academic Publishers, Dordrecht, 2001. 
[12] B. N. Sadovskiǔ, Limit-compact and condensing operators, Russian Math. Surveys 27 (1972), 85-155.

[13] R. C. Sine, On nonlinear contraction semigroups in sup norm spaces, Nonlinear Anal. 3 (1979), no. 6, 885-890.

[14] P. M. Soardi, Existence of fixed points of nonexpansive mappings in certain Banach lattices, Proc. Amer. Math. Soc. 73 (1979), no. 1, 25-29.

[15] S. Szufla, On the application of measure of noncompactness to existence theorems, Rend. Sem. Mat. Univ. Padova 75 (1986), 1-14.

Dariusz Bugajewski: Faculty of Mathematics and Computer Science, Adam Mickiewicz University, Matejki 48/49, 60-769 Poznań, Poland

E-mail address: ddbb@main.amu.edu.pl

Rafael Espínola: Departmento de Análisis Matemático, Facultad de Matematicas, Universidad de Sevilla, P.O. Box 1160, 41080 Sevilla, Spain

E-mail address: espinola@us.es 


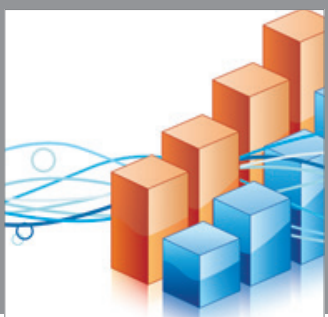

Advances in

Operations Research

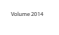

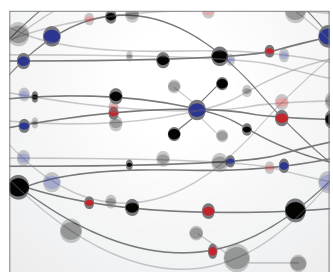

\section{The Scientific} World Journal
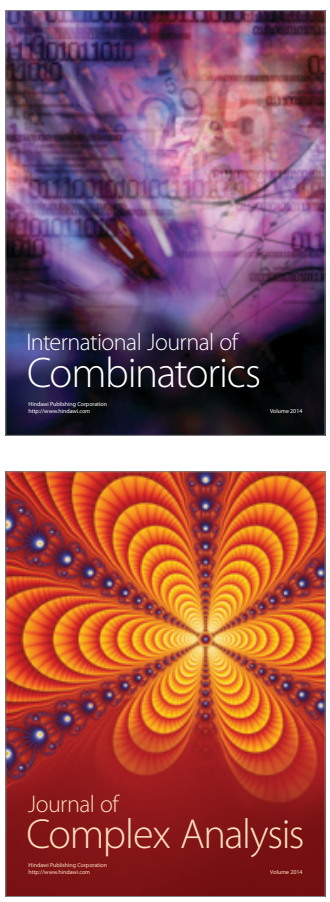

International Journal of

Mathematics and

Mathematical

Sciences
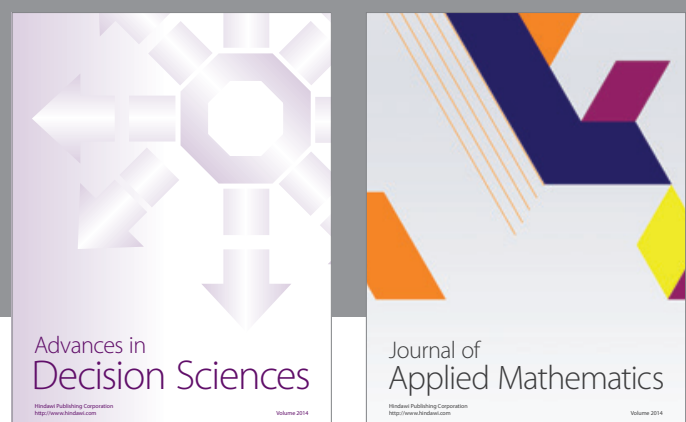

Journal of

Applied Mathematics
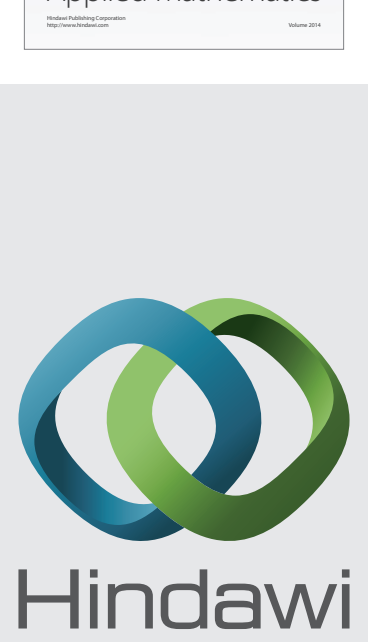

Submit your manuscripts at http://www.hindawi.com
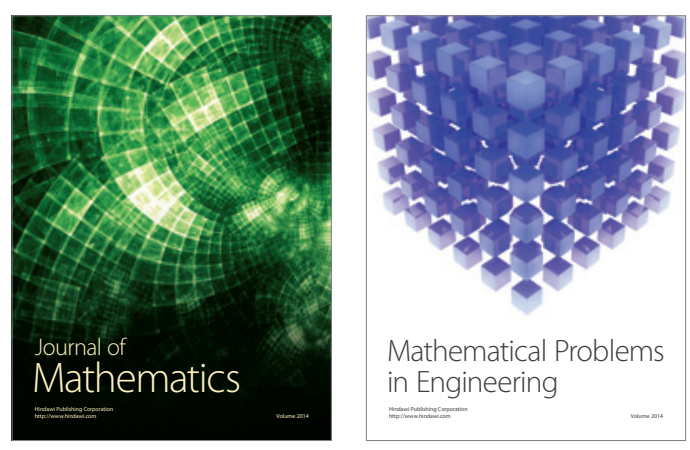

Mathematical Problems in Engineering
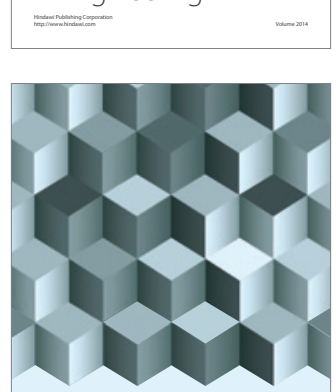

Journal of

Function Spaces
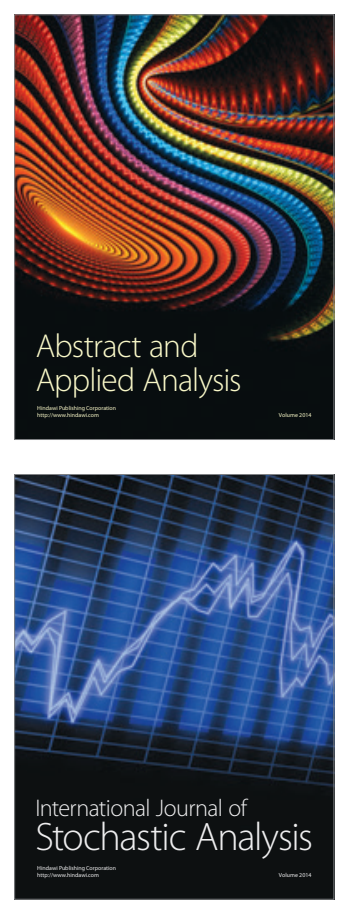

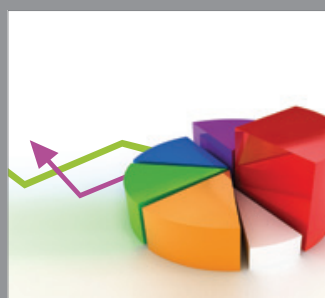

ournal of

Probability and Statistics

Promensencen
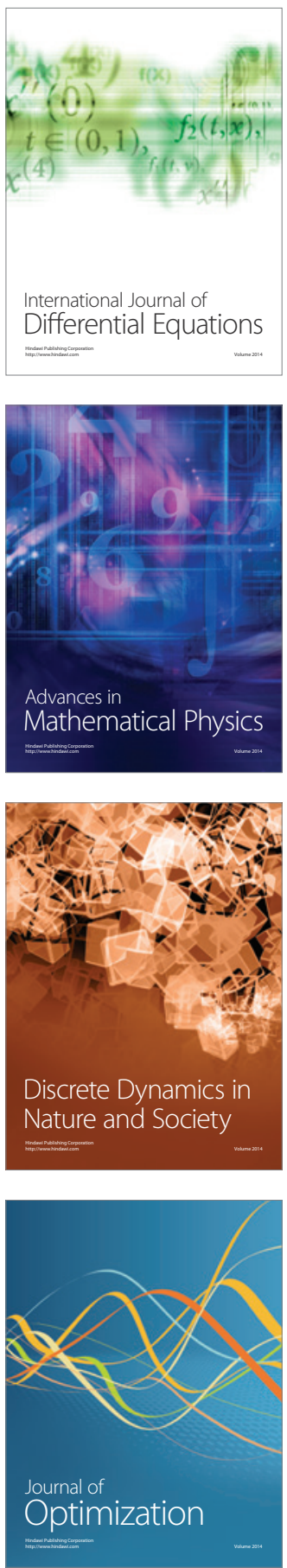